\title{
Antithèse et contextualisation : le débat sur la torture pendant la guerre d'Algérie (analyse d'un corpus de presse écrite, avril-décembre 1957)
}

Antithesis and Contextualization: The Debate about Torture during the Algerian War (Analysis of a Written Press Corpus, April-December 1957)

\section{Paola Païssa}

\section{OpenEdition}

Journals

Édition électronique

URL : http://journals.openedition.org/pratiques/2431

DOI : 10.4000/pratiques.2431

ISSN : 2425-2042

Éditeur

Centre de recherche sur les médiations (CREM)

\section{Référence électronique}

Paola Païssa, «Antithèse et contextualisation : le débat sur la torture pendant la guerre d'Algérie (analyse d'un corpus de presse écrite, avril-décembre 1957) », Pratiques [En ligne], 165-166 | 2015, mis en ligne le 01 octobre 2015, consulté le 10 décembre 2020. URL : http://journals.openedition.org/ pratiques/2431; DOI : https://doi.org/10.4000/pratiques.2431

Ce document a été généré automatiquement le 10 décembre 2020.

(C) Tous droits réservés 


\section{Antithèse et contextualisation : le débat sur la torture pendant la guerre d'Algérie (analyse d'un corpus de presse écrite, avril- décembre 1957)}

Antithesis and Contextualization: The Debate about Torture during the Algerian War (Analysis of a Written Press Corpus, April-December 1957)

Paola Païssa

1 Malgré son statut catégoriel flottant entre les figures «de pensée » et les figures «de construction ${ }^{1}$, l'antithèse est une configuration affichant une grande stabilité définitoire ${ }^{2}$, qui entretient des relations avec le contexte dont la tradition rhétorique a depuis toujours tenu compte. C'est par rapport à cet acquis que se déploiera notre réflexion. Nous appuyant sur un corpus de presse très vaste et correspondant à une question extrêmement controversée, nous observerons différents paliers de contextualité figurale, pour aboutir au concept plus dynamique de "contextualisation", préconisé par L. Gaudin-Bordes et G. Salvan (2013). Après une introduction consacrée à mettre en lumière quelques éléments $\mathrm{du}$ traitement traditionnel de l'antithèse, nous nous concentrerons sur une approche textuelle et pragma-énonciative de son fonctionnement dans le corpus choisi.

\section{Introduction : l'antithèse et le contexte dans le traitement classique de la figure}

Trois éléments saillants se dégagent des définitions de l'antithèse, qui représentent les caractéristiques intrinsèques de cette figure : le binarisme, l'opposition et le parallélisme des constituants. Le binarisme et l'opposition, ainsi que leur double possibilité de se 
manifester, sont réunis de manière très élégante dans la définition de P. Fontanier (1968 : 379) : «L'antithèse oppose deux objets l'un à l'autre, en les considérant sous un rapport commun, ou un objet à lui-même, en le considérant sous deux rapports contraires. » Le parallélisme, quant à lui, est élevé au rang de trait définitoire à la fin de l'article, lorsque P. Fontanier met en garde contre le risque de "prendre pour Antithèse toute façon quelconque d'exprimer une opposition d'idées", alors que cette figure exige "que les tournures se correspondent en opposant les idées " (Fontanier, ibid. : 381). La symétrie des parties, la correspondance des éléments mis en relation oppositive sont autant de traits systématiquement associés dans les définitions de l'antithèse, tant anciennes que modernes. Ces dernières insistent, en plus, sur la solidarité sémantique et pragmatique des composants : sur le plan sémantique, M. Bonhomme (1998:47) mentionne la nécessité que les éléments soient " situés sur le même axe sémantique ", faisant écho à l'idée de la concordance isotopique exprimée par le Groupe $\mu$ (1970); sur le plan pragmatique, on ponctue généralement l'effet de mise en relief d'une partie par rapport à l'autre (Morier, 1989) ou la fonction de renforcement de "l'idée principale », dérivant soit du « choc des oppositions » (Molinié, 1992: 57 ; Dupriez, $1984: 57$ ), soit de l'attraction réciproque des " polarités anti-orientées qui s'aimantent mutuellement » (Bonhomme, $2005: 66)^{3}$. Quant aux moyens formels d'actualiser le bipolarisme constitutif de la figure, les répertoires font état de plusieurs combinaisons, qui sous-entendent des liens syntaxiques fort différents ${ }^{4}$ : la juxtaposition $(A, B)$; la double exclusion (ni A, ni B) ; la contre-orientation argumentative (A, mais $B)$, l'opposition diachronique (A puis $B$ ), etc. De cette pluralité de constructions possibles, il en résulte que l'antithèse, à la différence de l'oxymore, figure de laquelle elle est souvent rapprochée ${ }^{5}$, peut s'appuyer soit sur une seule prédication (comme dans l'exemple hugolien «Foule triste, joyeuse » (emprunté à Bonhomme, 2010) soit, plus fréquemment, sur une prédication double, comme cela arrive dans la totalité des exemples de notre corpus. Dans un cas comme dans l'autre, au binarisme de la configuration correspond, au niveau énonciatif, la mise en scène d'un conflit de point de vue (dorénavant PDV) (Rabatel, 2008). Cette dimension ressortira clairement de notre analyse du corpus, ne comportant que des antithèses à double noyau prédicatif, caractérisées par une véritable théâtralisation de la divergence des PDV. La variété formelle qui marque le binarisme et le parallélisme concerne également l'opposition, qui peut s'actualiser suivant des procédés différents : au niveau lexical, la figure comporte la présence d'antonymes (comme dans l'exemple cité in: Fontanier, 1968:380, «Le ciel est dans ses yeux, l'enfer est dans son cœur ${ }^{6}$ ") ; au niveau morphosyntaxique, la configuration pivote sur des formes grammaticales inverses (généralement le singulier vs le pluriel, ou l'affirmation vs la négation, comme dans l'énoncé "Vous saviez tous les livres; vous ne saviez pas l'amour ", cité in: Bonhomme, 1998 : 47). Enfin, au niveau discursif, une tension dichotomique peut s'établir sous la pression du contexte, comme l'a souligné C. Fromilhague (1995). Outre les relations linguistiques, traditionnellement privilégiées dans le traitement de la figure, $C$. Fromilhague a mis en évidence la fonction du contexte discursif, moyennant le vers d'Hugo "Il voit, quand les peuples végètent ", choisi pour illustrer ce qu'elle appelle les « antonymes purement contextuels» (Fromilhague, 1995 : 50), c'est-à-dire les situations dans lesquelles l'antinomie n'est que le produit de la construction discursive (dans le célèbre poème d'Hugo "La fonction du poète ", l'antithèse voir/végéter se comprend sur le fond du contraste établi entre les gens du commun et le Poète, l'homme ayant «les pieds ici, les yeux ailleurs»). À ce niveau, constituant un premier cas de mise en avant du rôle du contexte dans le traitement de la configuration, nous utiliserons dorénavant la désignation "antithèse discursive ». De 
façon plus systématique, l'évocation du contexte se retrouve, dans les répertoires classiques, lorsqu'il est question de l'extension de l'antithèse. En effet, on a reconnu, dès l'Antiquité, une étendue extrêmement variable à cette configuration, ce qui correspond à autant de degrés différents de contextualisation. Au moins trois paliers, représentant l'empan de la figure, peuvent être identifiés :

- l'empan local correspond à l'actualisation occasionnelle d'un énoncé antithétique ponctuel, pouvant entretenir des relations plus ou moins fortes avec le cotexte discursif;

- l'empan textuel équivaut au cas fréquent où l'antithèse est reconnue comme principe organisateur d'un texte entier : il s'agit là d'une caractéristique que soulignent H. Morier (1989), C. Fromilhague (1995), ainsi que M. Bonhomme, qui mentionne « plusieurs sonnets de Du Bellay " comme exemples de constructions entièrement basées sur des réseaux antithétiques (Bonhomme, $1998: 47$ );

- l'empan macrotextuel se rapporte à la prise en compte de l'antithèse en tant qu'archétype, matrice génétique d'un ouvrage entier, présidant à la répartition des rôles ou des sujets et jouant donc, globalement, une fonction diégétique ou thématique. Ce statut de principe structurant potentiel, que l'antithèse partage avec d'autres figures, comme la métaphore ou la litote, permet de la considérer en tant que fondement d'ouvrages aussi divers que, par exemple, Le Rouge et le Noir, Les Rayons et les Ombres, Germinal, etc.

3 Répondant au caractère foncièrement disjonctif de notre système cognitif et de notre organisation anthropologique ( $c f$. Klinkenberg, 2000) et représentant «un puissant organisateur de la perception du monde et des discours » (Paveau, 2006: 205), l'antithèse est ouverte à la possibilité d'être saisie dans une contextualité maximale, touchant aux représentations psychologiques, sociales et à l'imaginaire artistique et sémiotique ${ }^{7}$. Comme nous le verrons, la question du contexte et de la contextualisation impose, de manière cruciale dans le cas du dispositif figural antithétique, une réflexion sur les limites supérieures de ces notions mêmes.

4 Toutes les caractéristiques que nous avons rappelées jusqu'ici expliquent un dernier élément récurrent, voire constant dans le traitement classique de l'antithèse: à la différence d'autres figures, il s'agit là d'une configuration fortement valorisée dans la tradition. Appartenant au paradigme des figures gorgianiques, l'antithèse a toujours été appréciée en tant que mécanisme typique du discours « habile» ou "brillant ${ }^{8}$, apte au style et aux genres élevés. Moins contrainte que d'autres figures inversives, comme le chiasme ou l'antimétabole (Rabatel, $2008: 22$ ), l'antithèse allie sa relative facilité à un rendement optimal, et ce à plusieurs égards : au point de vue du logos, elle est par excellence la figure de l'ordre, susceptible d'exploiter toute la séduction du pseudologique, en ce qu'elle s'appuie sur le lieu des contraires (si A est B, alors non A est non B) et qu'elle ambitionne, par là, à s'offrir comme un principe irréfutable; au point de vue du pathos, l'antithèse facilite l'adhésion émotive de l'énonciataire, à cause du potentiel esthétique et poétique que provoque la disjonction symétrique des parties (Plantin, 1993:492) ; au point de vue de l'éthos, cette configuration, comme d'autres figures oppositives, dessine l'image d'un locuteur-énonciateur « maitre de sa parole et de son vouloir dire » (Rabatel, 2008 : 31). À son extrême rentabilité discursive s'ajoute, en outre, son aptitude prononcée à la mémorisation: l'antithèse est traditionnellement la figure des aphorismes et des maximes ${ }^{9}$, candidate à engendrer ces formes d'énoncés mémorables, dont la communication médiatique est de plus en plus friande ${ }^{10}$. Enfin, grâce à la synergie de toutes ces raisons, l'antithèse est à l'évidence une des figures fondamentales du discours agonique (Angenot, 1982), dont peut relever le débat 
journalistique. De manière plus marquée que d'autres figures, elle présuppose la présence des conditions nécessaires à l'exercice même de la rhétorique : d'un côté l'existence d'un problème, d'un questionnement et, de l'autre, un « public dubitatif » et un « interdiscours contradictoire » (Charaudeau \& Maingueneau, 2002).

\section{Le corpus et la question du contexte}

5 Le corpus que nous allons analyser réunit au premier chef les aspects que nous venons d'évoquer, car il correspond à une conjoncture historique de "faible refoulement problématologique ", pour emprunter les termes que M. Meyer (1986) applique à la philosophie, c'est-à-dire un moment où l'interdiscours est traversé par des positionnements adverses et des clivages déchirants. En effet, il s'agit du débat sur l'emploi de la torture pendant la guerre d'Algérie, une question longtemps occultée et généralement ignorée, à l'époque, de l'opinion publique française, qui éclate dans la presse en avril 1957, obligeant le pouvoir en place à instituer, pour la première fois, une "Commission de sauvegarde des droits et libertés individuels d'initiative gouvernementale $\aleph^{11}$. Le rapport de cette commission, remis au gouvernement en juillet, soit un mois après la publication du compte rendu des travaux parallèles de la «Commission internationale contre le régime concentrationnaire » (CICRC) de la CroixRouge, est publié par Le Monde le 14 décembre 1957. Ce courageux scoop journalistique contraint le gouvernement, dès le lendemain, à abandonner sa stratégie de temporisation et à procéder à la publication officielle du document. Le dossier de presse que nous avons dépouillé, consultable auprès de l'Institut d'études politiques de Lyon ${ }^{12}$, couvre justement la période relative à ce moment discursif (Moirand, 2007), soit avril-décembre 1957. La problématique relative à la légitimité des moyens violents de répression embraye, pendant cette période, sur la question fondamentale du bien-fondé de la guerre et se double d'une interrogation sollicitant les fondements mêmes de la République, douze ans à peine après la conclusion de l'expérience de Vichy : la patrie des droits de l'homme est mise en cause pour l'adoption de méthodes comparables à celles des nazis, le devoir de dénoncer les excès policiers fait front à la censure imposée aux journaux, en Algérie autant qu'en métropole, alimentant une discussion sur la liberté de la presse et de l'information. Dans ce débat s'affrontent, comme dans une nouvelle affaire Dreyfus, deux conceptions de l'honneur de la France et s'installe un antagonisme de type néomaurassien, dressant les « patriotes » contre les « anti-France »"

6 À l'intérieur de ce cadre historique, la fréquence élevée, dans la presse écrite, d'une figure comme l'antithèse n'a certainement pas de quoi surprendre, car elle représente l'élément constitutif de l'argumentation éristique se déployant dans cette querelle. Or, c'est justement ce caractère constitutif qui rend difficile de saisir la relation de cette figure avec le contexte, car les limites de sa contextualisation potentielle sont « inexorablement repoussées vers l'infinitude » pour emprunter les mots que S. Moirand (1999 : 146) utilise pour le problème, de type divers mais comparable, de la délimitation des corpus médiatiques. En plus, la notion même de contexte est d'un maniement délicat, considérant sa nature complexe et fuyante : comme le rappelle F. Rastier (1998: 97), il s'agit d'un concept «souvent invoqué au lieu d'être défini » qui, de surcroit, « recouvre des réalités différentes en fonction du courant adopté » (Magaud, 2013 : 161). Deux questions posent tout spécialement problème dans la réflexion qui nous occupe. En premier lieu, l'épaisseur du contexte et de ses limites supérieures, face au déploiement 
d'une figure qui entretient des relations avec plusieurs couches de sens, interagissant sans cesse entre elles, depuis la strate la plus extérieure (le contexte sociohistorique, avec ses antinomies constitutives), jusqu'à la couche la plus infime et voilée du déploiement discursif. En deuxième lieu, la constatation qu'au moins deux approches devraient coexister dans notre travail, puisque les deux sont pertinentes ${ }^{14}:$ d'un côté, une approche textuelle, renouant avec une tradition scientifique qui, comme nous l'avons vu, avait déjà reconnu le rapport multidimensionnel de l'antithèse avec le contexte comme un trait figural spécifique de ce dispositif; de l'autre, une approche pragma-énonciative qui, en envisageant la figure essentiellement comme un marqueur dialogique (Gaudin-Bordes \& Salvan, 2012), tiendrait compte, à la fois, du dialogisme constitutif et du dialogisme montré qui s'y expriment. Tout en étant consciente de l'interaction constante de ces différents plans, nous comptons donc, pour les besoins de notre analyse, opérer une coupe horizontale de la notion de contexte et articuler notre investigation sur plusieurs niveaux. Suivent donc deux paragraphes : dans le premier, nous considérerons le rapport de la figure avec la contextualité maximale (contexte interdiscursiflarge), afin de montrer la facilité avec laquelle les oppositions sociohistoriques et politiques de l'époque se figent dans l'interdiscours en réseaux d'antithèses (dialogisme constitutif); dans le deuxième, nous prendrons en compte, en revanche, le contexte intradiscursif. Cet ensemble sera saisi dans sa double dimension : i) la singularité du dispositif discursif représenté par chaque article appartenant au corpus (cotexte $\left.e^{15}\right)$; ii) l'intertextualité du corpus, présentant de fréquents renvois explicites d'un article à l'autre. Notre approche du contexte intradiscursif se fera à deux niveaux : au niveau énonciatif, nous essaierons d'élucider la fonction que revêt l'antithèse pour déterminer le positionnement de l'énonciateur dans l'échiquier des points de vue en confrontation, ainsi que la posture d'appropriation ou de réfutation que déterminent, auprès de l'énonciateur, certains cas d'antithèses rapportées (dialogisme montré) ; au niveau textuel, nous mettrons en lumière le rôle d'organisateur discursif et d'outil de cohésion que joue la figure.

\section{Antithèses et contexte interdiscursif large}

7 Faute de pouvoir évoquer, en quelques mots, un cadre historique et un moment discursif aussi tourmenté que celui d'avril-décembre 1957, nous choisissons d'exemplifier, au moyen de quelques énoncés antithétiques prélevés dans notre corpus, les principales dichotomies dramatiques traversant l'époque. Les antithèses actualisées dans les exemples retenus auront ici une fonction d'illustration, certes non exhaustive, du contexte interdiscursif général et des enjeux oppositifs qui le traversent : elles montreront comment un dialogisme constitutif foncièrement contradictoire trouve un point de culmination, voire de coagulation dans des antithèses. Dans ce premier paragraphe, nous ne nous attarderons donc pas à considérer le cotexte des segments reportés et notre traitement sera délibérément schématique, l'ampleur du sujet exigeant des coupes sévères :

1 - « Peyrega est un martyr en deçà de l'eau et un coupable au delà » (Combat, 15 avril $1957)^{16}$.

2 - «On supporte désormais difficilement les réserves et les réticences opposées ici aux mesures de police et de répression prises là-bas par le gouvernement " ( L'Intransigeant, 21 avril 1957).

3 - « Il est plus difficile de peser moralement ses actes quand on vit sous la menace 
constante du terrorisme que lorsqu'on est à distance " (lettre des collègues de Peyrega, publiée dans Le Monde, 19 avril 1957)

Les trois premiers exemples montrent l'opposition la plus radicale, c'est-à-dire la déchirure territoriale France-Algérie: derrière la fiction de l'«Algérie française " se trouvant à l'origine de la guerre ( $c f$. Maldidier, 1971), se profile nettement, dans le discours de 1957, le clivage qui sépare de manière irrémédiable deux manières de sentir et de vivre le conflit. Pour la métropole, la guerre d'Algérie est, après l'Indochine, le prototype des guerres modernes, lointaines et vécues principalement à travers les médias; par contre, pour les Français d'Algérie, le conflit est une réalité quotidienne, impliquant de front leur territoire ${ }^{17}$. Dans les exemples 1 et 2 , les couples de déictiques antithétiques en deçà/au delà et ici/là-bas sous-entendent de fortes tensions discursives : dans l'énoncé 1, s'affrontent deux qualificatifs à orientation axiologique inverse (martyr) coupable) appliqués à l'auteur d'une dénonciation de violences policières commises contre les musulmans ${ }^{18}$ (M. Peyrega, doyen de la Faculté de Droit d'Alger ${ }^{19}$ ); dans l'énoncé 2 , le journal de droite L'Intransigeant se fait l'interprète des difficultés qu'éprouve la communauté française d'Algérie à comprendre les griefs que certains milieux intellectuels de la métropole adressent au régime répressif mis en place « là-bas » par le gouvernement. La différence entre la position morale d'une collectivité exposée directement aux dangers du terrorisme, par rapport à une autre vivant la paix et la prospérité de la fin des années 1950, devient explicite dans l'exemple 3, qui propose l'antithèse discursive vivre sous la menace/être à distance.

9 Une autre opposition concerne la question centrale de la légitimité de la torture. La presse, surtout celle d'inspiration catholique, rapporte et reformule souvent la position officielle qu'ont assumé, le 14 mars 1957, les cardinaux et archevêques de France :

4 - « Il n'est pas légitime de mettre au service d'une cause, même bonne, des moyens intrinsèquement mauvais " (La Gazette de Lausanne, 14 avril 1957).

Si cette position réussit, à travers l'antithèse bon /mauvais, le tour antimachiavélique de prendre des distances avec les «moyens", sans condamner leur finalité, elle se double souvent d'une interrogation concernant l'ampleur du phénomène, avec l'intention de le minimiser ou, à l'opposé, de le généraliser. On s'interroge donc sur la systématicité vs l'exceptionnalité de la torture et sur la conscience qu'en ont les différents acteurs en jeu (politique nationale, administration locale, police coloniale, armée). Là aussi on enregistre des opinions situées aux antipodes, culminant dans des antithèses, comme dans ce témoignage de l'envoyé spécial de Combat, Maurice Clavel :

5 - «Je sais qu'il y a eu des sévices. Je crois qu'il y a eu des tortures. Je ne crois pas qu'il y ait eu un "univers de la torture". Il y a eu des tortures moins que l'on ne crie, plus que l'on n'avoue. [...]. Je crois qu'ils (officiers supérieurs, résistants, Compagnons de la Libération, anciens déportés) ont consciemment refusé de connaître ce qui se passait dans leurs unités [...]. Je crois que l'horrible phénomène de la torture a créé chez des êtres honnêtes ce sentiment nouveau, ambigu : bonne foi dans la mauvaise foi (ou l'inverse), très différent de la duplicité, de l'hypocrisie, et qui demande des appréciations morales nouvelles: en tout cas ni peccadille ni crime. Je sais comment des professeurs de morale peuvent devenir coquins de vertu » (Combat , 23 avril 1957).

11 Exploitant toutes les ressources de la " critique ni-ni » (" ni peccadille ni crime »), dont R. Barthes (1970: 144-146) a révélé la fausse neutralité idéologique, l'envoyé de Combat tranche également sur une autre opposition fondamentale, celle concernant l'opportunité de taire vs dénoncer la pratique de la torture: sur la question, il affiche, à travers une 
antithèse fondée sur la double exclusion, une équidistance inspirée d'une tartuferie qui devait être assez commune à l'époque :

6 - «Ce qu'il ne fallait pas faire : une campagne. [...] Il ne fallait ni étaler ni cacher $»^{20}($ Combat, 23 avril 1957).

12 La polarisation silence vs devoir de dénonciation est une autre contradiction traversant foncièrement l'interdiscours, susceptible de se concrétiser également dans des formulations antithétiques :

7 - «L'institution de la Commission d'enquête prouve déjà que nous avons eu raison de crier sur les toits ce que l'on ne voulait même pas laisser murmurer dans les antichambres " (France Observateur, 11 avril 1957).

8 - « Notre vieux peuple [...] a plus besoin de vérités cruelles que de lâche silence » ( Demain, 28 novembre 1957).

13 Dans l'exemple 7, tiré de l'article "Le silence est de sang ", paru dans un numéro de France Observateur qui sera saisi par la censure, l'ancien déporté et Compagnon de la Libération Claude Bourdet, l'un des premiers à avoir dénoncé le recours à la torture et payé de la prison ses courageuses accusations ${ }^{21}$, se réjouit d'avoir eu raison de «crier sur les toits » une vérité qu'on ne voulait même pas "laisser murmurer dans les antichambres ». La même dichotomie vérités vs silence, enrichie des axiologiques antagoniques cruelles/ lâche est proposée dans l'exemple 8, puisé dans un article signé par le général Billotte, l'un des rares militaires français à avoir critiqué la méthode de «la question», en polémique ouverte avec le général Massu' ${ }^{22}$.

\section{Antithèse et contexte intradiscursif}

Dans ce paragraphe, nous prendrons en considération: dans un premier temps, la fonction de la figure dans la construction du cotexte discursif de quelques articles appartenant au corpus et, dans un second temps, la présence d'antithèses dans le tissu des renvois intertextuels caractérisant l'ensemble de notre corpus.

\subsection{Antithèse et cotexte discursif : image de l'énonciateur}

15 À ce niveau, il est question de montrer les modalités par lesquelles l'antithèse interagit avec la texture pragma-énonciative du discours et contribue à définir le positionnement de l'énonciateur. Comme nous l'avons expliqué en introduction, notre corpus ne comporte que des antithèses à double prédication: dans la mise en scène des PDV divergents que joue cette figure (Rabatel, 2008), chaque prédication relève d'un PDV1 réfutant le PDV2. Ce conflit de PDV peut relever et participer à la construction de différentes images de l'énonciateur. Suivant la taxinomie de la topique énonciative d'A. Rabatel (2005) nous en avons repéré fondamentalement deux : la posture de coénonciation et la posture de surénonciation.

Dans le premier cas, l'antithèse est non seulement fonctionnelle à la proposition d'une médiation entre les deux PDV divergents, mais elle en représente le point d'orgue, comme on peut le constater dans l'exemple suivant :

9 - «En fin de compte, dans notre pays, après les déchirements que l'on souhaite éviter, la victoire n'en resterait pas moins à ceux qui, contre tout, persistent à croire au respect de l'homme. De nouveaux colonel Picquart et Emile Zola, même condamnés, seraient vainqueurs » (Combat, 11 avril 1957). 
(a) Combat annonce la constitution, en avril 1957, de la Commission d'enquête sur la torture et présente cet événement comme la solution du contraste des positions sur la question de la torture : le rétablissement de la vérité auquel donnera lieu cette initiative gouvernementale est posé dans le discours comme un fait certain, qui fournira une issue aux affrontements. L'antithèse discursive condamnés/vainqueurs mobilise la mémoire interdiscursive relative à l'affaire Dreyfus, évoquée à travers deux personnages que le jugement de l'histoire a reconnu comme vainqueurs, bien qu'ils aient été auparavant condamnés. Les antonomases discursives ${ }^{23}$ "De nouveaux colonel Picquart et Emile Zola », se rattachent à l'idée, largement développée dans le texte, qu'il faut sortir de l'«équivoque » et surmonter la "coupure", comparable à celle qui avait divisé les Français à la fin du XIX ${ }^{\mathrm{e}}$ siècle : ces antonomases soutiennent l'antithèse médiatrice, qui se fonde également sur une sorte de court-circuit métaleptique contrefactuel (ceux qui, aujourd'hui, sont condamnés pour avoir dénoncé, pourraient, demain, être vainqueurs). L'emploi de connecteurs concessifs comme « en fin de compte », l'usage d'un « on » de connivence («déchirements que l’on souhaite éviter»), l'emploi du conditionnel («la victoire n'en resterait pas moins...») sont autant de traces de la construction de la posture de coénonciation qui débouche dans l'antithèse-médiation.

La posture de surénonciation, dans laquelle l'énonciateur essaie d'imposer son PDV, parvient également à son sommet dans l'antithèse, qui est utilisée comme un moyen de boucler la boucle discursive :

10 - «Un innocent auquel on donne une paire de gifles est torturé. Un poseur de bombes auquel on donne une paire de gifles est-il torturé ?» (Combat, 22 novembre 1957).

L'exemple 10 est tiré d'une intervention du général Massu publiée dans Combat. Comme il le fera plus tard en termes explicites ${ }^{24}$, Massu ne nie pas l'existence de la torture : en revanche, il choisit de revendiquer son utilité, son "efficacité ». Et, pour rendre son discours plus convaincant, il met en doute la notion de torture elle-même (par ailleurs désignée par l'euphémisme métonymique « une paire de gifles »), s'interrogeant sur les limites à partir desquelles on peut parler de torture. À travers l'antithèse discursive ( innocent / poseur de bombes), déployée dans la forme d'une interrogation rhétorique ${ }^{25}$, il essaie d'imposer une nouvelle doxa qui considère la torture comme un moyen admissible. Et, à la fin de l'article, c'est à travers une autre antithèse qu'il arrive à renverser complètement le principe fondamental de la Déclaration des droits de l'homme de 1948 et le PDV du droit international : alors que celui-ci affirme l'égalité des droits de l'innocent et du coupable, le général Massu finit par conclure que :

11 - «Les droits de l'innocent sont supérieurs aux droits du coupable» (Combat, 22 novembre 1957).

La posture de surénonciation, le «je dis » s'imposant sur le " on dit », dont les indices sont foisonnants dans le texte (assertions impératives, interrogations pressantes, etc.), culmine dans cette antithèse, qui constitue évidemment une affirmation fausse. Les caractéristiques argumentatives et esthétiques de l'antithèse, que nous avons évoquées dans l'introduction (la séduction pseudologique de l'opposition, la symétrie et la correspondance des parties, etc.), sont ici mises au service d'un forçage de la parole qui se présente, de surcroit, avec l'allure d'une maxime d'autorité, destinée à se figer en énoncé mémorable. 


\subsection{Antithèse et contexte interdiscursif exhibé}

21 Il nous reste à examiner les rapports qu'établit la figure de l'antithèse au niveau de l'ensemble du corpus, c'est-à-dire dans le réseau de ses renvois intertextuels. Nous analyserons donc quelques cas d'antithèses relevant du dialogisme montré, car elles proviennent d'un énonciateur second (E2) explicitement inscrit dans le texte. Les antithèses rapportées sont fréquentes dans les articles, où elles assument la fonction d'un véritable argument, par rapport auquel tout le discours de l'énonciateur primaire (E1) prend position: la facilité de figement aphoristique de l'antithèse facilite ce jeu de reprise.

Un premier exemple nous est offert par un article fort polémique de l'intellectuel catholique Joseph Rovan qui constitue une réponse à l'opposition établie par le journaliste démocrate-chrétien Louis Terrenoire, dans un article paru dans Carrefour, le 3 avril 1957 :

12 - «Contre la torture ? Oui ! Contre la France ? Non!» (Témoignage Chrétien, 12 avril 1957).

Toute l'argumentation de Rovan représente une réfutation de cette antithèse qui, d'après lui, serait fondée sur une généralisation arbitraire, ressuscitant l'esprit maurassien des antiFrance : tous ceux qui critiquent la torture seraient des traitres, ayant mis sur pied un « complot » contre la France. Dans cet article, l'antithèse de E2 fournit le point de départ du discours de E1 et une antithèse rapportée en constitue aussi l'aboutissement. En effet, à la fin de l'article, un deuxième énoncé antithétique, au statut formulaire, est avancé comme argument à l'appui de la position de E1 : une citation de Renan joue le rôle d'un argument d'autorité, pour soutenir la légitimité des critiques, surtout si elles proviennent d'autres intellectuels catholiques, comme François Mauriac ou Pierre-Henri Simon :

13 - «Le mot de Renan: "Périsse la France pourvu que l'esprit vive" peut être retourné contre les chrétiens à la Terrenoire [qui] excusent l'inexcusable » ( Témoignage Chrétien, 12 avril 1957).

Les déchirements des milieux catholiques sont encore plus intensément représentés dans un dernier exemple, qui trouve sa place ici, parce qu'il prend appui sur un renvoi intertextuel et qu'il est entièrement construit sur une suite d'antithèses. Cet article nous permettra donc d'illustrer, suivant une approche textuelle, comment cette figure peut jouer le rôle d'organisateur discursif et d'outil de cohésion du texte. Il s'agit là du degré de contextualisation de l'antithèse qui a déjà été mis en évidence dans le traitement scientifique de cette figure, notamment par M. Bonhomme (2005:66), qui prend en compte l'antithèse parmi les figures fonctionnant comme des « opérateurs périodiques $\mathrm{du}$ discours".

L'article en question constitue une réfutation, signée par Gilbert Cesbron, de l'argumentation proposée par l'aumônier parachutiste en faveur de la possibilité d'admettre "un interrogatoire sans sadisme mais efficace », publiée dans Le Monde du 30 juin 1957. L'" efficacité ", comme l'a déjà montré le discours de Massu examiné plus haut, était l'argument le plus puissant parmi ceux avancés en faveur d'une légitimation de la torture : il se présentait comme une médiation à l'opposition humanité / cruauté. Or, la réponse de Cesbron se base justement sur une mise en cause du concept d'« efficacité » : l'article repose entièrement sur des antithèses, précisément parce qu'il procède à un démontage de la médiation apparente que constitue la notion d'efficacité et 
au rétablissement des oppositions irréductibles qu'elle fait semblant d'escamoter. C'est surtout l'opposition réalistes vs idéalistes qui est mise à contribution, pour : i) tourner en dérision, grâce à un «mouvement d'exagération absurdifiante » (Plantin, 1993: 493), la formule proposée par l'aumônier («Un interrogatoire sans sadisme mais efficace... Un pogrom sans haine mais efficace... Un camp de déportation où le règlement serait humain mais efficace »); ii) pour dénoncer le prix trop élevé qu'a coûté «l'œuvre efficace» d'Hitler, Staline, Mussolini et même du "vieux maréchal», considérant la fin que l'histoire a réservée à ces personnages :

14 - «C'est le destin des réalistes quand l'humiliante loi du pendule ne vient pas leur opposer à temps ces irritants "idéalistes" qui leur paraissent compromettre si fort les résultats obtenus, alors qu'ils les humanisent seulement et s'efforcent [...] de rendre respirable un monde trop "efficace" » (Le Monde, 5 juillet 1957).

Par ailleurs, ce qui fait scandale pour un écrivain catholique comme Gilbert Cesbron, c'est que ces propos inadmissibles viennent d'un chrétien, comme l'aumônier parachutiste, un "loup» se cachant "sous la cape du berger", qui est accusé ici d'opérer des manipulations et des assimilations abusives de distinctions capitales, qu'il faut garder à tout prix :

15 - «Le rôle des chrétiens est précisément, depuis deux mille ans, de proclamer un certain nombre de principes particulièrement "inefficaces" et de se tenir prêts à mourir pour eux. C'est - pour parler un langage laïque - le rôle des idéalistes; et leur duel (ou leur alternance) avec les réalistes est sans doute le moindre mal, en attendant le royaume de Dieu. Mais il ne faut pas fausser le jeu, monsieur l'aumônier, ni que les uns tiennent le langage des autres »...(Le Monde, 5 juillet 1957).

\section{Conclusions}

La figure de l'antithèse interagit avec le contexte à plusieurs niveaux et suivant les multiples dimensions de celui-ci. Cependant, deux directions nous paraissent fondamentales, qui rejoignent la notion de contextualisation, telle que la définissent L. Gaudin-Bordes et G. Salvan (2013: 20) :

«Appliquée aux figures du discours, la contextualisation englobe le travail réciproque du contexte sur la figure (le contexte est nécessaire pour interpréter une figure) et de la figure sur le contexte (la figure a, à des degrés variables, un effet retour sur le contexte).»

C'est là une conception dynamique de la figure du discours, qui la saisit dans une relation à double entrée, comme figure contextualisée et, en même temps, contextualisante. Or, l'antithèse, telle que nous l'avons décrite dans cette étude, relève pleinement de ce double mouvement de contextualisation. Avant tout, cette figure est un point de condensation, une sorte de précipité dans lequel se figent les données appartenant au dialogisme constitutif, c'est-à-dire au contexte interdiscursif le plus étendu : à cet égard, notre étude a largement confirmé une caractéristique qui avait été depuis toujours reconnue à cette figure. En deuxième lieu, notre observation du corpus a montré le rôle actif que joue l'antithèse dans la mise en discours d'une réalité fortement dichotomisée comme celle du débat sur la torture en 1957: au niveau pragma-énonciatif, la figure constitue l'aboutissement du processus de construction des postures d'énonciation; au niveau textuel, elle fonctionne comme un organisateur du déploiement discursif ; enfin, au niveau intertextuel, l'antithèse participe directement du jeu des reprises en écho qui caractérise inévitablement la controverse. De manière plus spécifique, un dispositif 
figural à fonction argumentative et au potentiel formulaire comme l'antithèse, montre bien la circularité du dire médiatique : une figure peut servir à boucler le dire et se trouver également à la source du dire. Comme le disait R. Barthes dans un article célèbre sur les maximes de La Rochefoucauld, l'antithèse «n'est au fond que le mécanisme tout nu du sens [...], et comme, dans toute société évoluée, le retour aux sources fonctionne finalement comme un spectacle surprenant, ainsi l'antithèse est devenue une pointe, c'està-dire le spectacle même du sens » (Barthes, 1972 : 79).

C'est finalement la synergie de la rhétorique et de l'analyse du discours qui s'avère à même de saisir tout le potentiel contextualisant de cette figure. À ce propos, des considérations touchant aussi le niveau de la dispositio, qui distingueraient fonction proactive et fonction réactive de la figure (en ouverture ou en clôture d'un texte), pourraient encore apporter des éléments utiles à la réflexion.

\section{BIBLIOGRAPHIE}

ADAM, J.-M., GRIZE, J.-B. \& ALI BOUACHA, M. (éds) (2004) : Texte et discours : catégories pour l'analyse, Dijon, Éditions universitaires de Dijon.

ADAM, J.-M. \& HEIDMANN U. (éds) (2005) : Sciences du texte et analyse du discours. Enjeux d'une interdisciplinarité, Genève, Slatkine.

ANGENOT, M. (1982) : La Parole pamphlétaire, Paris, Payot.

BARTHES, R. (1970) [1957] : « La critique Ni... Ni », in : R. Barthes, Mythologies, Paris, Éd. Le Seuil, p. 134-137.

- (1972) [1961] : « Préface à La Rochefoucauld, Réflexions ou Sentences et Maximes », in :

R. Barthes, Le Degré zéro de l'écriture suivi de Nouveaux essais critiques, Paris, Éd. Le Seuil, p. 69-88.

воNномме, M. (1998) : Les Figures clés du discours, Paris, Éd. Le Seuil.

- (2005) : Pragmatique des figures du discours, Paris, H. Champion.

- (2010) : «La rhétorique des figures entre formalisme et énonciation », in : S. Badir \&

M. G. Dondero (coords), « Le Groupe $\mu$ entre rhétorique et sémiotique », Protée, 38, 1, p. 65-74.

- (2012) : «Polyphonie et ethos dans l'interrogation rhétorique : le cas des Fables de La

Fontaine », in : F. Calas, C. Fromilhague, A.-M. Garagnon \& L. Susini (éds), Les Figures à l'épreuve du discours, Paris, Presses de l'Université Paris-Sorbonne, p. 39-52.

BRANCHE, R. (1999) : « La Commission de sauvegarde pendant la guerre d'Algérie. Chronique d'un échec annoncé ", Vingtième Siècle. Revue d'histoire, 61, p. 14-29.

- (2005) : La Guerre d'Algérie : une histoire apaisée ?, Paris, Éd. Le Seuil.

Charaudeau, P. \& maingueneau, D. (éds) (2002) : Dictionnaire d'analyse du discours, Paris, Éd. Le Seuil.

DUMARSAIS, C. (1988) [1730] : Des tropes, Paris, Flammarion.

DUPRIEZ, B. (1984) : Gradus. Les Procédés littéraires, Paris, 10/18.

FONTANIER, P. (1968) [1827] : Les Figures du discours, Paris, Flammarion. 
FROMilhague, C. (1995) : Les Figures de style, Paris, A. Colin.

GAUDIN-BORDES, L. \& SALVAN, G. (2012) : « La figure est-elle un marqueur dialogique ? », in : F. Calas, C. Fromilhague, A.-M. Garagnon \& L. Susini (éds), Les Figures à l'épreuve du discours, Paris, Presses de l'Université Paris-Sorbonne, p. 115-124.

GAUDIN-BORDES, L. \& SALVAN, G. (2013) : « Contextualisation et hyperpertinence figurale », in : G. Salvan (éd.), « Figures et contexte(s) », Le Discours et la langue, t. 4.2, p. 17-24.

GIRARDET, R. (1960) : « Pouvoir civil et pouvoir militaire dans la France contemporaine », Revue française de sciences politiques, 1, p. 5-38.

GROUPE M (1970) : Rhétorique générale, Paris, Larousse.

KESSEL, P. (2003) : Guerre d'Algérie : écrits censurés, saisis, refusés, 1956-1960-1961, Paris, Éd. L'Harmattan.

KLINKENBERG, J.-M. (2000) : « L'argumentation dans la figure », Cahiers de praxématique, 35, p. 59-86. KRIEG-PLANQUe, A. \& OlLIVIER-YANIV, C. (éds) (2011) : « Les "petites phrases" en politique ", Communication \& langages, 168.

LEROY, S. (2000) : "Quels fonctionnements discursifs pour l'antonomase du nom propre? ", Cahiers de praxématique, 35, p. 87-113.

MALDIDIER, D. (1971) : « Le discours politique de la guerre d'Algérie : approche synchronique et diachronique », Langages, 23, p. 57-86

MAGAUD, V. (2013) : « La syllepse au service d'un genre : le cas du discours de campagne présidentielle ", Le discours et la langue, t. 4.2, p. 161-171.

MEYER, M. (1986) : De la problématologie : philosophie, science et langage, Bruxelles, Mardaga.

MOIRAND, S. (1999) : «Les indices dialogiques de contextualisation dans la presse ordinaire », Cahiers de praxématique, 33, p. 145-184.

- (2007), Les Discours de la presse quotidienne, Paris, Presses universitaires de France.

MolinIÉ, G. (1992) : Dictionnaire de rhétorique, Paris, Le Livre de Poche.

MORIER, H. (1989) [1961] : Dictionnaire de poétique et de rhétorique, Paris, Presses universitaires de France.

MONTE, M. (2008) : « Le jeu de points de vue dans l'oxymore: polémique ou reformulation? », Langue française, 160, p. 37-54.

PAïsSA, P. (2013) : « Le silence sur la torture pendant la guerre d'Algérie », Mots. Les langages du politique, 103, p. 39-54.

PAVEAU, M.-A. (2006) : Les prédiscours. Sens, mémoire, cognition, Paris, Presses Sorbonne Nouvelle.

PLANTIN, C. (1993) : « Lieux communs dans l'interaction argumentative », in : C. Plantin (éd.), Lieux communs, Paris, Kimé, p. 480-496.

RABATEL, A. (2005) : «Les postures énonciatives dans la co-construction dialogique des points de vue : coénonciation, surénonciation, sousénonciation », in : J. Bres, P.-P. Haillet, S. Mellet, H. Nolke \& L. Rosier (éds), Dialogisme, polyphonie: approches linguistiques, Bruxelles, Duculot, p. 95-110.

- (éd) (2008a) : « Figures et point de vue », Langue française, 160.

- (2008b) : « Points de vue en confrontation dans les antimétaboles PLUS et MOINS », Langue française, 160, p. 21-36. 
RASTIER, F. (1998) : «Le problème épistémologique du contexte et le statut de l'interprétation dans les sciences du langage ", Langages, 129, p. 97-111.

ROBRIEUX, J.-J. (1998) : Les Figures de style et de rhétorique, Paris, Dunod.

ROCHE, E. (2007) : Étude des discours de presse écrite française sur la violence et la torture pendant la guerre d'Algérie - Le Monde, L'Humanité, Le Figaro, L'Express, France Observateur, 1954-1962, thèse, Université Lumière Lyon 2.

\section{NOTES}

1. À l'évidence, la figure a pu être rangée dans un groupe ou dans l'autre suivant les critères généraux des classifications et selon que l'accent est mis sur l'effet pragmasémantique de l'opposition ou sur la disposition syntaxique des constituants. Traditionnellement, la figure est classée parmi les figures dites «de pensée», ou «métalogismes", ou figures «macrostructurales» (cf. Dumarsais, 1988 ; Fontanier, 1968 ; Groupe $\mu, 1970$; Molinié, 1992) ; à l'intérieur de classements divers et plus articulés, elle a pu être répertoriée comme une «figure de construction» (Fromilhague, 1995; Robrieux, 1998) ou une «figure syntaxique par opposition » (Bonhomme, $1998: 47)$.

2. Les définitions pivotent en général sur les trois éléments que nous reprenons dans notre introduction. Le Groupe $\mu$ s'en différencie, préférant pointer l'idée du métalogisme par répétition (au lieu d'énoncer A, elle ajoute : A n'est pas non-A) (Groupe $\mu, 1970: 121$ ).

3. C'est précisément l'exploitation de cette attraction qui permet parfois de formuler de fausses antithèses, comme le montre ce titre italien, paru dans un journal proche de Silvio Berlusconi : «Silvio sotto processo e i teppisti in libertà » («Silvio en procès et les voyous en liberté », Libero, 24/10/13). Aucun rapport n'existait en réalité entre les affaires judiciaires de Berlusconi et les quelques jeunes vandales mis en liberté à ce moment-là : le rapprochement qu'opère le titre obtient cependant un effet pragmatique, ne tenant qu'à l'opposition apparente qu'il met en place. Il s'agit d'un cas d'antithèse manipulatoire, dont nous n'avons pas trouvé d'exemples dans notre corpus.

4. Cette question mériterait une étude spécifique qui, à ma connaissance, fait encore défaut.

5. Cf. M. Bonhomme (2010), qui rejoint sur cela la position exprimée par le Groupe $\mu$ (1970:121), ayant rangé l'antithèse parmi les métalogismes et l'oxymore parmi les métasémèmes. Sur les différences des deux figures, voir aussi M. Monte (2008).

6. P. Fontanier (1968 : 380) puise l'exemple dans l'Henriade de Voltaire, décrivant ainsi « la tendre Hypocrisie aux yeux pleins de douceur ».

7. L'évocation, de la part de P. Fontanier, des contrastes de tons dans la peinture et la musique, pour mieux comprendre l'effet de la figure dans le discours, est fort significative à cet égard (Fontanier, 1968 : 379).

8. Cette caractéristique, soulignée déjà par le Pseudo-Hermogène, se retrouve chez P. Fontanier (1968 : 379).

9. Cf. les réflexions de R. Barthes (1972) sur la fonction de l'antithèse dans les maximes de F. de La Rochefoucauld.

10. Que l'on songe, par exemple, au phénomène des "petites phrases ", tel qu'il est décrit dans A. Krieg-Planque et C. Ollivier-Yaniv (2011).

11. Sur les questions relatives au silence sur la torture, à l'inefficacité des dénonciations antérieures à cette période et aux raisons qui ont conduit, au cours de l'année 1957, à une rupture de la «spirale du silence ", nous renvoyons à la thèse de E. Roche (2007) et à P. Païssa 
(2013). Quant au travail de la Commission de sauvegarde et à la polémique qui a accompagné dans la presse la publication tardive de son rapport, voir R. Branche (1999).

12. En ligne: http://doc.sciencespo-lyon.fr/Ressources/Documents/DocEnLigne/Algerie/ documents-torture.html._Pour le détail des quotidiens, de toute tendance politique, qui figurent dans le dossier, nous renvoyons à P. Païssa (2013).

13. Les mémoires interdiscursives concernant l'affaire Dreyfus, Maurras et l'expérience de la collaboration sont constamment mobilisées dans ce débat, avec de nombreuses références explicites.

14. Par ailleurs, l'articulation de la dimension textuelle avec la discursive s'impose, notamment après les travaux de J.-M. Adam $(2004,2005)$.

15. Par cotexte nous entendons donc ici, suivant l'emploi usuel, l'environnement immédiat des occurrences figurales à l'intérieur d'un texte, c'est-à-dire un article appartenant au corpus, pris dans sa singularité. En revanche, ce que nous appelons «contexte intradiscursif» est un ensemble plus large, comprenant aussi les liens intertextuels internes au corpus.

16. Dans les exemples, sauf avis contraire, c'est nous qui soulignons les termes mis en opposition. 17. Sur le clivage entre armée et population civile et entre Algérie et métropole pendant la guerre coloniale, il est intéressant de voir ce qu'écrivait, dès 1960, l'historien R. Girardet (1960).

18. Nous gardons les désignations utilisées à l'époque, car selon nous une modernisation altérerait l'authenticité des documents sur lesquels s'appuie notre recherche. Sur la question des modalités d'appellation des événements et des acteurs de la guerre d'Algérie par les chercheurs contemporains, voir R. Branche (2005: 349 et suiv.).

19. Le doyen Peyrega a démissionné en signe de protestation contre le comportement violent de la police : son geste est fort apprécié par les collègues et les intellectuels de la métropole et, à l'inverse, est sévèrement blâmé par les collègues et les étudiants français d'Alger.

20. Le segment se présente en italique dans le texte.

21. Claude Bourdet publie, dès 1951, dans L'Observateur, « Y a-t-il une Gestapo en Algérie ? »; en 1955 parait son célèbre "Votre Gestapo d'Algérie ». Il est incarcéré le 31 mars 1956 pour « entreprise de démoralisation de l'armée ».

22. Notre corpus comprend différents échanges publiés dans les journaux entre les généraux Billotte et Massu, dans les mêmes mois où le général De Bollardière est incarcéré pour ses critiques à l'armée. On peut trouver d'autres traces de l'opposition entre Billotte et Massu chez P. Kessel (2003: 157).

23. Nous empruntons cette appellation à S. Leroy (2000).

24. Dès 1971, le général Massu publie La Vraie Bataille d'Alger, où il admet l'emploi de la torture ; lors de la reprise du débat sur la question en 2000, il déclare son regret d'avoir eu recours à ces pratiques (Le Monde, 23 novembre 2000).

25. Pour une illustration du pouvoir assertif et manipulatoire sous-jacent à la figure de l'interrogation rhétorique, $c f$. M. Bonhomme (2012).

\section{RÉSUMÉS}

L'étude décrit le rapport existant entre l'antithèse et le contexte dans le traitement traditionnel de la figure et dans un corpus de presse relatif au débat sur l'emploi de la torture pendant la guerre d'Algérie (avril-décembre 1957). Après avoir analysé le contexte interdiscursif large et les 
couples antithétiques qui le traversent, l'article se concentre sur le « contexte intradiscursif »: au niveau pragma-énonciatif, il montre que la figure contribue à construire l'image de l'énonciateur et qu'elle participe du tissu des reprises intertextuelles internes au corpus; au niveau textuel, il illustre le rôle d'organisateur discursif que joue le dispositif antithétique. Enfin, l'étude met en lumière la fonction de l'antithèse dans le processus dynamique de la «contextualisation", c'est-à-dire dans le double mouvement d'une configuration à la fois "contextualisée » et "contextualisante ", saisie dans la situation particulière $d u$ "discours agonique » caractérisant la discussion sur le recours à la torture pendant la guerre d'Algérie, dans la presse écrite de l'époque.

The present study analyzes the relation between antithesis and context by looking at the traditional use of this figure of speech and a corpus concerning the debate about the use of torture in Algeria between April and December 1957. After taking the wider inter-discursive context into account, including the main antithetical pairs therein, the study focuses on the "intra-discursive context". A pragma-enunciative approach is adopted to analyze how the figure of speech contributes to constructing the enunciator's image and the network of intertextual references within the corpus. In addition, the role of discourse organizer played by the figure of speech will be highlighted from a textual perspective. Finally, emphasis will be put on the fundamental function of antithesis in the dynamic process of "contextualization", both as a contextualized and as a contextualizing figure, with particular reference to the situation of "discours agonique" which characterizes the debate in the press of that time about the use of torture in the Algerian War.

INDEX

Keywords : antithesis, context, contextualization, torture, Algerian War Mots-clés : antithèse, contexte, contextualisation, torture, guerre d'Algérie

\section{AUTEUR}

\section{PAOLA PAIISSA}

Université de Turin (Italie) 